\section{ORIGINAL} RESEARCH

\section{J.S. Anderson}

H.S. Dhatt

M.A. Ferguson

M. Lopez-Larson

L.E. Schrock

P.A. House

D. Yurgelun-Todd

\title{
Functional Connectivity Targeting for Deep Brain Stimulation in Essential Tremor
}

BACKGROUND AND PURPOSE: Deep brain stimulation of the thalamus has become a valuable treatment for medication-refractory essential tremor, but current targeting provides only a limited ability to account for individual anatomic variability. We examined whether functional connectivity measurements among the motor cortex, superior cerebellum, and thalamus would allow discrimination of precise targets useful for image guidance of neurostimulator placement.

MATERIALS AND METHODS: Resting BOLD images (8 minutes) were obtained in 58 healthy adolescent and adult volunteers. Regions of interest were identified from an anatomic atlas and a finger movement task in each subject in the primary motor cortex and motor activation region of the bilateral superior cerebellum. Correlation was measured in the time series of each thalamic voxel with the 4 seeds. An analogous procedure was performed on a single subject imaged for 10 hours to constrain the time needed for single-subject optimization of thalamic targets.

RESULTS: Mean connectivity images from 58 subjects showed precisely localized targets within the expected location of the ventral intermediate nucleus of the thalamus, within a single voxel of currently used deep brain stimulation anatomic targets. These targets could be mapped with single-voxel accuracy in a single subject with 3 hours of imaging time, though targets were reproduced in different locations for the individual than for the group averages.

CONCLUSIONS: Interindividual variability likely exists in optimal placement for thalamic deep brain stimulation targeting of the cerebellar thalamus for essential tremor. Individualized thalamic targets can be precisely estimated for image guidance with sufficient imaging time.

ABBREVIATIONS: $\mathrm{AAL}=$ Automated Anatomical Labeling; $\mathrm{BOLD}=$ blood oxygen level-dependent; $\mathrm{DTI}=$ diffusion tensor imaging; $\mathrm{ET}=$ essential tremor; $\mathrm{fMRI}=$ functional $\mathrm{MR}$ imaging; $\mathrm{M} 1=$ primary motor cortex; $\mathrm{MNI}=$ Montreal Neurologic Institute; MPRAGE = magnetization-prepared rapid acquisition of gradient echo; SPM = statistical parametric mapping; $\mathrm{VIM}=$ ventral intermediate nucleus of the thalamus

D eep brain stimulation, a technique consisting of depth electrode placement in deep gray nuclei, has allowed successful treatment of patients with medication-refractory essential tremor, ${ }^{1}$ with fewer adverse effects than with thalamotomy. ${ }^{2}$ Typically, results have been achieved by placing depth electrodes within the Vim or cerebellar thalamus. ${ }^{1}$

While anatomic atlas-based localization of the VIM has been traditionally used, the direct method of electrode placement based on MR imaging coordinates may provide better targeting results as shown previously for globus pallidus internus localization for dystonia. ${ }^{3}$ However, targeting the VIM is problematic for image guidance because thalamic subnuclei do not show demarcation of boundaries on standard MR im-

Received February 13, 2011; accepted after revision March 8.

From the Division of Neuroradiology (J.S.A., H.S.D.) and Departments of Psychiatry (M.L.-L.), Neurology (L.E.S.), and Neurosurgery (P.A.H.), University of Utah School of Medicine, Salt Lake City, Utah; and the Brain Institute (J.S.A., M.L.-L., L.E.S., P.A.H., D.Y.-T.) and Department of Bioengineering (J.S.A., M.A.F.), University of Utah, Salt Lake City, Utah.

This work was supported by National Institutes of Health grants K08 MH092697 (J.S.A.) and R01 DA020269 (D.Y.-T.) and by the Ben B. and Iris M. Margolis Foundation (J.S.A.).

Paper previously presented at: Annual Meeting of the American Society of Neuroradiology, June 4-9, 2011; Seattle, Washington.

Please address correspondence to Jeffrey S. Anderson, MD, Department of Neuroradiology, University of Utah 1A71 School of Medicine, Salt Lake City, UT 84132; e-mail: andersonjeffs@gmail.com

Indicates open access to non-subscribers at www.ajnr.org

http://dx.doi.org/10.3174/ajnr.A2638 aging sequences. Postplacement outcome analysis based on the brain atlas by Schaltenbrand and Wahren ${ }^{4}$ and the diagrams of Taren et $\mathrm{al}^{5}$ for thalamic targeting has found that electrode location with the most effective clinical outcome was just anterior to the VIM and $3 \mathrm{~mm}$ from the anterior border of main sensory nucleus ventralis caudalis. ${ }^{6}$ In a study with $>2$ years of follow-up in 37 subjects, optimal lead placement was reported to be $12.3 \mathrm{~mm}$ lateral to midline and $6.3 \mathrm{~mm}$ anterior to the posterior commissure in the plane of the anterior and posterior commissures. ${ }^{6}$

The lack of direct image guidance is particularly important, given that even minimal variation in lead placement may result in long-term clinical failure or less than satisfactory treatment of essential tremor. ${ }^{7}$ Long-term failure rates for deep brain stimulation in essential tremor have been reported to be $13 \%-40 \%$, due to a hypothesized physiologic tolerance or suboptimal lead placement. ${ }^{8-12}$ In 1 study, even as little as a 2 -mm error in placement resulted in only a $17 \%$ chance of producing essential tremor control defined by criteria of $>66 \%$ improvement in tremors. ${ }^{6}$

A recent study by Yamada et $\mathrm{al}^{13}$ showed that the VIM could be approximated by using diffusion tensor tractography, providing a novel way to accurately image the VIM without relying on anatomic atlases. Using directional information provided by DTI for tractography and anatomic knowledge that cerebellothalamocortical tract dentate projections intersect the spinothalamic tracts within the VIM, the authors were able to define landmarks for in vivo localization of the VIM. 
Yet this technique yielded approximations that were more lateral than those typically used by anatomic landmarks and did not identify the more medial regions of the VIM. The authors suggested that given the current technical artifacts associated with DTI techniques, the procedure may not be suited to individualized targeting. ${ }^{13}$

Cytoarchitectonic studies by Morel et $\mathrm{al}^{14}$ have demonstrated interindividual differences in the location and size of thalamic nuclei that cannot be easily taken into account by standardization procedures. Furthermore, cortical connectivity parcellation of thalamic nuclei by using diffusion tensor tractography in healthy individuals reveals that there is both quantitative and qualitative variation in probabilistic thalamic atlases due to individual variability in precise volumes and location of borders of different nuclei. ${ }^{15}$ Interindividual variations in connectivity-defined parcellations reflect the difficulty in precisely matching variations in brain and thalamic sizes and shapes in registration of images across groups.

As an alternative for individualized preoperative image guidance, we attempted to identify the ventral intermediate nucleus by performing functional connectivity MR imaging measurements of thalamic connectivity to cerebellar and motor cortical brain regions. Functional connectivity uses synchrony of task or resting-state fMRI time-series data to estimate, quantitatively, correlation between 2 brain regions. ${ }^{16,17}$ Such an approach has been used previously to define differential thalamocortical connectivity within the thalamus ${ }^{18-20}$ and has allowed precise identification of subtle differences in connectivity between adjacent voxels in other functional brain regions. $^{21}$

\section{Materials and Methods}

\section{Subject Characteristics}

BOLD fMRI data were obtained from 59 healthy adolescent and adult volunteers, examined after informed consent in accordance with procedures approved by the University of Utah institutional review board (mean age, $18.0 \pm 4.9$ years; age range, 11-35 years; 32 males, 26 females). Data from these subjects have been previously reported. ${ }^{21-23}$ All subjects had no Diagnostic and Statistical Manual of Mental Disorders-IV Axis I diagnoses based on a diagnostic semistructured psychiatric interview and screening surveys as previously described. ${ }^{22}$

Additionally, one hundred 5-minute scans were obtained during 10 imaging sessions (10 scans per session) on 1 of the subjects (male, 39 years of age) during a 3-week period. Five of the sessions were performed while the subject was instructed to "keep your eyes open and remain awake" and 5 of the sessions were performed while the subject was watching ten 5-minute clips from Bugs Bunny cartoons (Looney Tunes Golden Collection, Volume 1; Warner Home Video, Hollywood, California). The same 10 clips were used for each of the 5 cartoon sessions in the same order, with the clips synchronized to the onset of the BOLD acquisition by a fiber optic trigger pulse. Images from this dataset have been previously reported in the context of the reproducibility of functional connectivity measurements throughout the brain. ${ }^{23}$

In all 59 subjects, an additional BOLD sequence was obtained consisting of a 4-minute block design in which the subject was instructed to alternately touch the thumbs with each of the secondthrough-fifth digits in turn for 20 seconds followed by 20 seconds of rest. Six such blocks were obtained, with visual cues "Task" and "Rest" to switch between finger movement task and rest blocks.

\section{Data Acquisition}

Images were acquired on a 3T Magnetom Trio (Siemens, Erlangen, Germany) scanner with a 12-channel head coil. The scanning protocol consisted of an initial 1-mm isotropic MPRAGE acquisition for an anatomic template. BOLD echo-planar images $(\mathrm{TR}=2.0$ seconds, $\mathrm{TE}=28 \mathrm{~ms}$, generalized autocalibrating partially parallel acquisition with acceleration factor $=2$, forty sections at $3-\mathrm{mm}$ section thickness, $64 \times 64$ matrix) were obtained during the resting state. The BOLD acquisition resolution was $3.0-\mathrm{mm}$ isotropic. Prospective motion correction was performed during BOLD imaging with a prospective acquisition-correction technique sequence. An 8-minute resting scan (240 volumes) was obtained for each of the group subjects. One hundred 5-minute scans ( 155 volumes) were obtained for the individual subject. An additional field map scan was obtained for each subject for distortion correction. For all BOLD sequences, simultaneous plethysmograph (pulse oximeter) and chest excursion (respiratory belt) waveforms were recorded for off-line analysis.

\section{fMRI Postprocessing}

Postprocessing of BOLD images has been previously described. ${ }^{22}$ Briefly, BOLD images were processed with RETROICOR ${ }^{24}$ by using the AFNI software package (http://afni.nimh.nih.gov/afni/) ${ }^{25}$; section-timing correction (SPM8, Wellcome Department of Imaging Neuroscience, London, United Kingdom); motion and distortion correction (realign and unwarp, SPM8); coregistration to MPRAGE (SPM8); segmentation of gray matter, white matter, and CSF (SPM8); normalization to the MNI template brain (SPM8, T1.nii); PSTCor (http://www.pstcorp.com/) ${ }^{22}$ allowing removal by regression of motion, physiologic, CSF, white matter, and soft-tissue signals; bandpass filtering between 0.001 and $0.1 \mathrm{~Hz}^{26}$; and linear detrend at each voxel in the brain.

\section{Region-of-Interest Selection}

Four regions of interest were used to define functional connectivity, located in bilateral M1 and bilateral motor activation regions of the superior cerebellum. In the group of 58 subjects, the mean time-series was extracted from left and right precentral gyrus clusters (M1) from an MNI-normalized version of the AAL atlas ${ }^{27}$ packaged with the WFU PickAtlas toolbox software (http://fmri.wfubmc.edu/downloads/WFU_ PickAtlas_User_Manual_v3.0.pdf). ${ }^{28}$ The regions of interest for superior cerebellar clusters were selected from group-level activation maps from 58 subjects by using second-level analysis in SPM with a standard general linear model after first extracting finger movement $>$ rest activation maps for each subject. A threshold of $\mathrm{T}>9$ was selected to ensure that superior cerebellar clusters were distinct, with no overlap and no voxels that extended across the midline.

The superior cerebellar and primary motor regions of interest for the individual subject were obtained from a general linear model analysis of the finger movement task for this subject, with a threshold of $\mathrm{T}>8$ for $\mathrm{M} 1$ clusters and a threshold of $\mathrm{T}>4$ for superior cerebellar clusters, with $>10$ voxels per cluster selected to achieve distinct clusters for connectivity seeds.

\section{Functional Connectivity Measurements}

A mask of the bilateral thalamus was obtained from the AAL atlas ${ }^{27}$ and was used to identify voxels within the thalamus. For each thalamic voxel in each subject, time-series data were extracted and a 

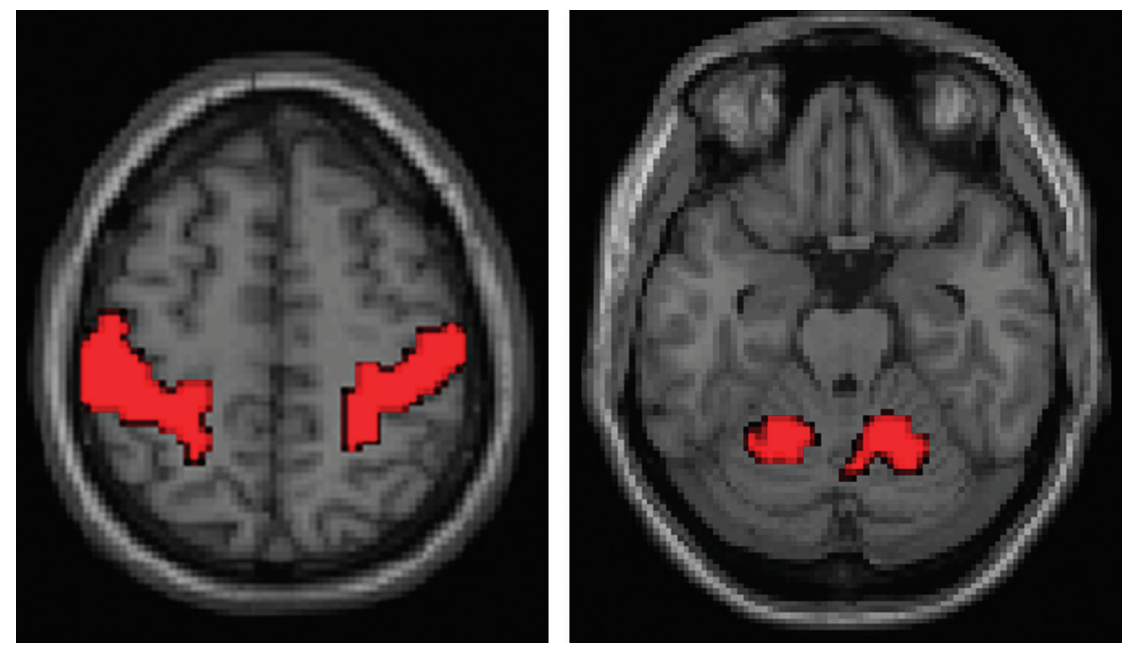

Fig 1. Seed regions of interest used for calculating thalamic motor functional connectivity in 58 subjects. Analogous regions of interest were derived from the finger movement task for the individual subject.

Pearson correlation coefficient was measured between each voxel and the time-series data from the 4 motor cortices and superior cerebellar seeds. Correlation values were Fisher-transformed for improved normality by evaluating the hyperbolic arctangent. ${ }^{29}$ The resulting $4 z$ scores were averaged for correlation measurements with each thalamic voxel and the 4 seed regions of interest to obtain a mean connectivity $z$ score for the voxel.

\section{Results}

We attempted to "triangulate" functional connectivity among the thalamus, motor activation regions of the superior cerebellum, and primary motor cortex by measuring the correlation in each voxel of the thalamus with the bilateral primary motor cortex and bilateral superior cerebellar motor areas. We used the same seed regions of interest for these 4 regions in 58 subjects, shown in Fig 1. The primary motor cortex (M1) regions of interest were defined by the AAL atlas, ${ }^{27}$ whereas the superior cerebellar regions of interest represented group-level activation from a bilateral finger movement task. In results for a single subject, analogous regions of interest were derived from activation in the bilateral M1 and bilateral superior cerebellum from the finger movement task.

Once the relevant region-of-interest masks were identified, Pearson correlation coefficients between each voxel in the thalamus and the regions of interest from Fig 1 were averaged, with the mean correlation from 58 subjects shown in Fig 2. The group correlation results show cylindric volumes in the bilateral thalamus that correspond to the expected location of the VIM, for which peak correlation to the seeds lies at MNI coordinates (left: $\mathrm{x}=-11, \mathrm{y}=-25, \mathrm{z}=2$; right: $\mathrm{x}=-13, \mathrm{y}=$ $-26, z=1$ ) within a $5-\mathrm{mm}$ distance from the coordinates used for thalamic deep brain stimulation based on anatomic land$\operatorname{marks}(\mathrm{x}= \pm 12.5, \mathrm{y}=22, \mathrm{z}=1)$.

When similar measurements of correlation to the primary motor and superior cerebellar cortices were obtained within a single subject, nearly identical results were obtained for peak correlation coordinates. This was true for data from grouped scanning sessions when the subject was in a resting state as well as for the correlations observed when the subject was watching cartoons. This result is reassuring in that it suggests that the motor correlation measured in the thalamus is not dependent on the specific task the subject was performing. Moreover, reproducible targets for thalamic brain stimulation are critical if a connectivity-based method is used for image guidance. Because precise coordinates will be required for thalamic targeting, likely requiring relatively long image acquisition times, it is helpful to establish that results can be obtained during a task that patients can easily perform for an extended period, such as watching a film.

The correlation peak seen in the VIM is not the only one seen in the individual's result. An additional peak is seen in the expected location of bilateral lateral geniculate nuclei, which might suggest that the motor regions also show specific connectivity to visual inputs necessary for coordination of movement with the subject's visual reference frame. Although the group-level and individual results are precise, there are notable differences between the targets suggested in Figs 2 and 3. This may indicate interindividual variation in the optimal location of motor network connectivity in the thalamus.

The individual subject results of Fig 3 were obtained from 5 hours of imaging time each. For an estimate of how much imaging time was necessary in this subject to obtain reproducible results, we examined results of a single scanning session (50 minutes BOLD imaging time) for this subject. In some of the scanning sessions, a clear peak was not identified that would suggest a target for deep brain stimulation. When groups of 2 scans were averaged (100 minutes BOLD imaging time), all 5 groups of 2 scans showed a peak in the region of the VIM, but the variability in the measurements was greater than a single voxel in location, not optimal for precise image guidance.

Results are shown in Fig 4 when 10 groups of 3 scanning sessions were averaged ( 150 minutes of BOLD imaging time). The sessions were selected randomly from among the 10 scanning sessions. In all 10 cases, a clear peak was obtained in close proximity to the targets identified by using all 10 sessions. The mean and SD of the targets obtained from the 10 groups of 3 scanning sessions were the following: left: $x=-10.8 \pm 1.1$, $y=-20.1 \pm 1.0, z=-0.5 \pm 1.0$; right: $x=4.6 \pm 1.3, y=$ $-20.6+ \pm 1.9, \mathrm{z}=0.0 \pm 1.1$. For the 10 groups of scans eval- 


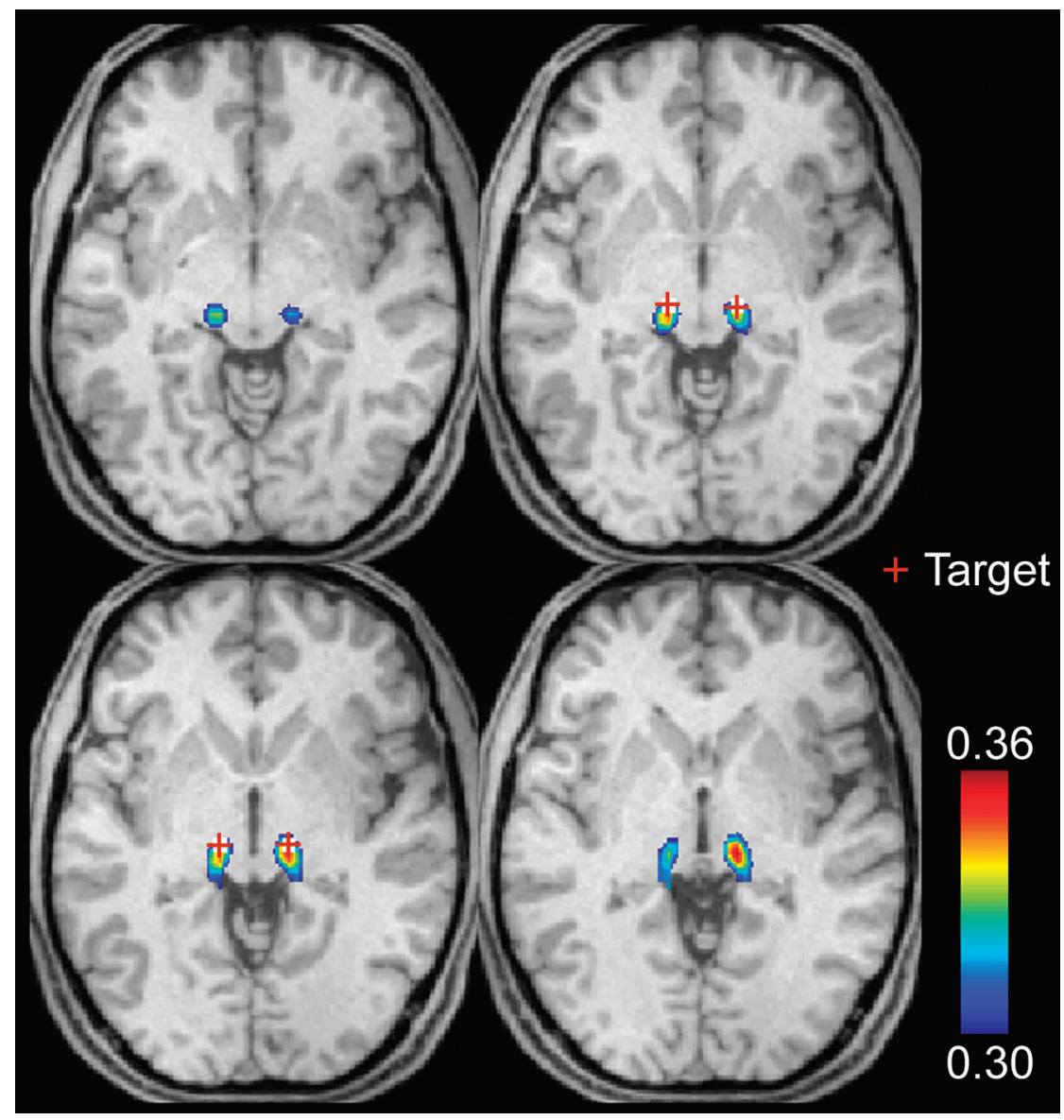

Fig 2. Thalamic motor functional connectivity averaged from 58 subjects. The red cross identifies the anatomic coordinates of the voxel used for deep brain stimulation. Images are in radiologic format. Section locations are $\mathrm{MNI}: \mathrm{z}=-2,0,2,4$ (top left to bottom right).

uated, the mean distance from the target by using 3 sessions compared with using all 10 sessions was $<2 \mathrm{~mm}$, less than the size of a single voxel. In all cases, the distance between the targets was $<5 \mathrm{~mm}$ among different groups of 3 scans.

\section{Discussion}

We have demonstrated that functional connectivity among the bilateral primary motor cortex, bilateral cerebellar motor cortex, and bilateral thalami is greatest in thalamic voxels located within $3 \mathrm{~mm}$ of clinically optimized targets for deep brain stimulation in essential tremor, validating these coordinates as representing the intersection of connections between the cerebellum and motor cortex in the thalamus. Additionally, we demonstrated the feasibility of identifying individualized coordinates within 1 subject with accuracy on the order of a single voxel. Given the known variability in the size and shape of the thalamus, as well as interindividual variations in thalamic nuclei location, ${ }^{14,15}$ it is possible that functional connectivity targeting may allow improved localization of deep brain stimulator targets over those obtained from conventional anatomic landmarks.

Reliability of functional connectivity measurements is directly related to imaging time. In a study assessing reliability as a function of imaging time, variability in connectivity metrics decreased with the square root of imaging time, with intersession correlation improving from 0.7 to 0.85 when 40 minutes of imaging time was used instead of 5 minutes. ${ }^{30}$ The same 1/square root $(n)$ relationship between test-retest reliability and imaging time has also been observed within a single individual, as well as for convergence to group means of measurements within a population of subjects. ${ }^{23}$ When average correlation strengths over an entire network are examined, correlation measurements stabilize after approximately 5 minutes of imaging time, reaching asymptotic values. ${ }^{30}$ Yet individual functional connectivity measurements, such as we obtained in comparing adjacent thalamic voxels, are noisier, and high-resolution thalamic targeting was only possible with imaging times in the neighborhood of 3 hours.

Deep brain stimulation targets for essential tremor have been chosen to target the "cerebellar thalamus" or location where cerebellar motor connections arrive in the thalamus; however, it remains unknown how deep brain stimulation is able to successfully mitigate tremor or what specific neurophysiologic changes are achieved during treatment in the cerebellothalamocortical circuitry. Although our imaging technique allows connection-based targeting, we do not know which connections are optimal, and comparison of deep brain stimulation outcomes with functional connectivity-based targets will be required to verify whether this approach can identify high-yield targets for neurostimulator placement. Even if the location our technique identifies is not optimal, the methods suggest a systematic approach to identifying targets by comparing brain connectivity with functional outcomes.

The resolution achieved in our results, with the target identi- 
Rest, Eyes Open

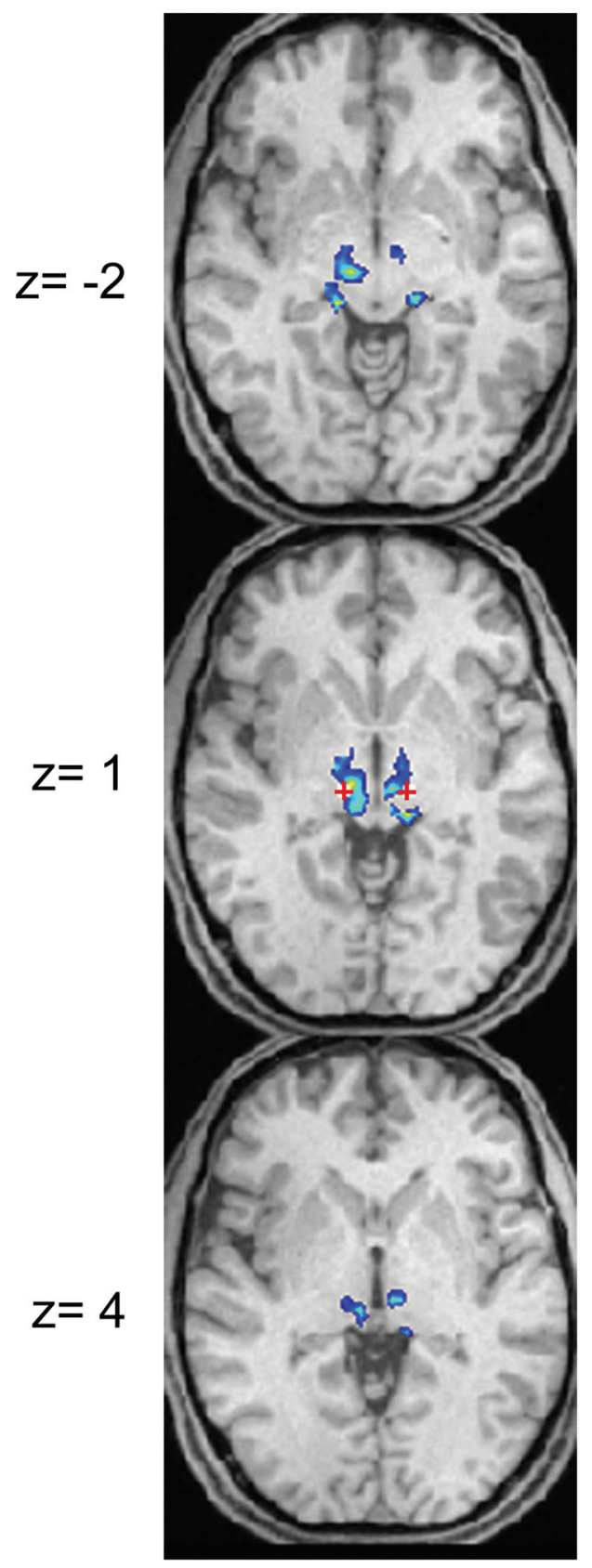

Watching Cartoons

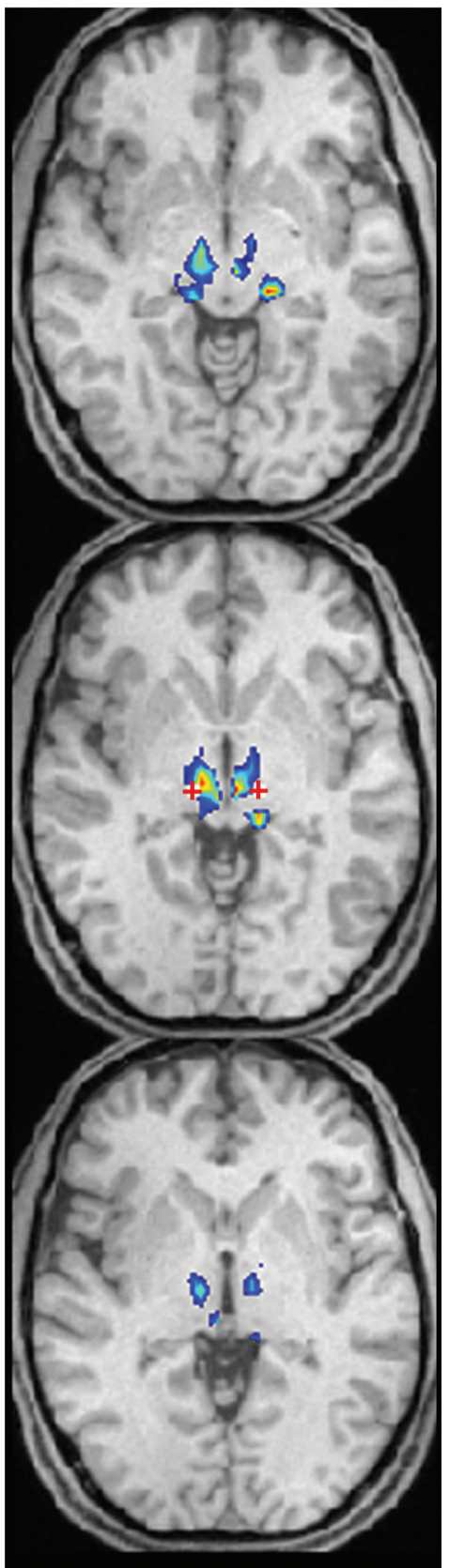

Fig 3. Single-subject thalamic motor functional connectivity. Voxels within the top $50 \%$ of peak $z$ scores are shown in the thalamus. The red cross indicates the voxel that would be targeted by using anatomic positioning for thalamic deep brain stimulation. The left column shows mean connectivity scores from 50 five-minute scans obtained during 5 sessions with the subject in a resting state, and the right column, from 50 five-minute scans obtained during 5 different sessions with the subject watching cartoons. Images are in radiologic format.

fied within 2-3 $\mathrm{mm}$, is on the order of the acquisition resolution and is the main reason for the long scanning times used in the individual subjects. It is possible to estimate BOLD targets at a resolution even finer than the sampling resolution if interpolation is used, particularly if numerous sequences are obtained, allowing meaningful differences in connectivity between neighboring voxels. In our data, the target reproducibility error was slightly smaller than the size of an individual voxel.

All of the subjects used in the above analysis had no tremor or other neurologic illness. It is possible that subjects with tremor may have altered cerebellothalamocortical connectiv- ity that may affect targeting for brain-stimulator placement. Moreover, reliability results were performed in a single subject. Other subjects may vary in the reproducibility of functional connectivity measurements. Furthermore, it is possible that when the technique is applied to older individuals, obtaining sufficient high-quality BOLD imaging data may be difficult, given patient motion or intolerable scanning durations for patients. To mitigate these challenges, we show that results obtained when the subject is watching a video clip are similar to those obtained in a resting-state acquisition and that data from multiple scanning sessions can be combined to obtain 


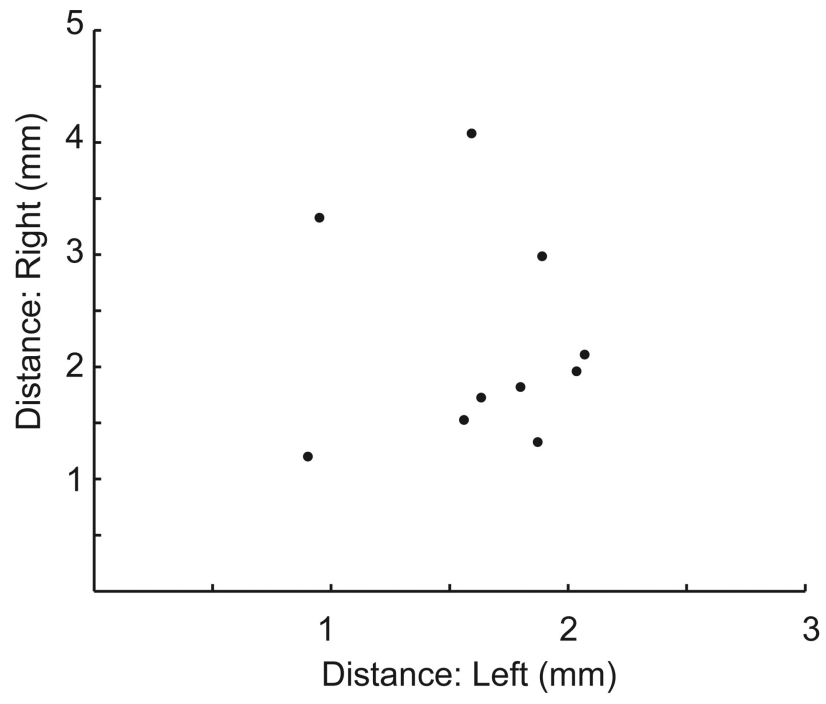

Fig 4. Variability in the target by using only 3 sessions. Ten different sets of 3 scanning sessions were randomly selected from the 10 scanning sessions. The optimal target (peak connectivity) was selected for the average of each group of 3 scans and was compared with the target by using all 10 scans. Distance from the target is shown for the left and right thalami for each of the 10 sets of 3 sessions.

sufficient resolution for identifying thalamic targets. In our experience, patient motion is mitigated when the subject is engaged in a task such as watching a video clip. This may have the additional benefit in reducing the chance that a subject will fall asleep during a protracted acquisition because it has been demonstrated that functional connectivity measurements can depend on cognitive state or task during acquisition. ${ }^{23}$

\section{Conclusions}

Functional connectivity measurements among the bilateral motor cortex and bilateral superior cerebellar motor regions and the thalamus show peak correlation within the thalamus across subjects within $3 \mathrm{~mm}$ of the targets currently used for deep brain stimulation in essential tremor, validating these coordinates as a location of cerebellar and motor cortical convergence in the thalamus. With sufficient imaging time, likely in the neighborhood of 3 hours of BOLD imaging, individual subject measurements can be reproducibly obtained with the precision of approximately $2 \mathrm{~mm}$, demonstrating feasibility for individualized targeting of deep brain stimulator placement. Further work is necessary to demonstrate the feasibility in patients with tremor and to assess the effects of functional connectivity targeting on clinical outcomes.

Disclosures: Lauren E. Schrock: Consultant. Teva, Details: Teva supports a scholarly journal club for neurology residents, which I lead a few times per year. Journal articles and topics discussed are chosen by the faculty member leading journal club. Honorarium is $\$ 500-800$ per session. Paul House: Consultant. Medtronic, Boston Scientific, Details: Consultant relationship with Boston Scientific. Surgical proctor relationship with Medtronic. Neither company was involved in any way with any of the submitted work. Deborah Yurgelun-Todd: Research Support (including provision of equipment or materials): Kyowa Hakko, Details: Kyowa Hakko provided grant funding and reagents to study the effects of supplementation with citicoline; Speaker Bureau: Kyowa Hakko, Details: I present research findings based on studies of citicoline for Kyowa Hakko.

\section{References}

1. Benabid AL, Pollak P, Gervason C, et al. Long-term suppression of tremor by chronic stimulation of the ventral intermediate thalamic nucleus. Lancet 1991;337:403-06
2. Tasker RR. Deep brain stimulation is preferable to thalamotomy for tremor suppression. Surg Neurol 1998;49:145-53, discussion 153-144

3. Vayssiere N, Hemm S, Cif L, et al. Comparison of atlas- and magnetic resonance imaging-based stereotactic targeting of the globus pallidus internus in the performance of deep brain stimulation for treatment of dystonia. J Neurosurg 2002;96:673-79

4. Schaltenbrand G, Wahren W. Atlas for Stereotaxy of the Human Brain. Stuttgart, Germany: Thieme Medical Publishers; 1977

5. Taren J, Guiot G, Derome P, et al. Hazards of stereotaxic thalamectomy: added safety factor in corroborating $\mathrm{x}$-ray target localization with neurophysiological methods. J Neurosurg 1968;29:173-82

6. Papavassiliou E, Rau G, Heath S, et al. Thalamic deep brain stimulation for essential tremor: relation of lead location to outcome. Neurosurgery 2008; 62(suppl 2):884-94

7. Pilitsis JG, Metman LV, Toleikis JR, et al. Factors involved in long-term efficacy of deep brain stimulation of the thalamus for essential tremor. J Neurosurg 2008;109:640-46

8. Benabid AL, Benazzouz A, Hoffmann D, et al. Long-term electrical inhibition of deep brain targets in movement disorders. Mov Disord 1998;13(suppl 3):119-25

9. Kumar R, Lozano AM, Sime E, et al. Long-term follow-up of thalamic deep brain stimulation for essential and parkinsonian tremor. Neurology 2003;61:1601-04

10. Koller WC, Lyons KE, Wilkinson SB, et al. Long-term safety and efficacy of unilateral deep brain stimulation of the thalamus in essential tremor. Mov Disord 2001;16:464-68

11. Hariz MI, Shamsgovara P, Johansson F, et al. Tolerance and tremor rebound following long-term chronic thalamic stimulation for Parkinsonian and essential tremor. Stereotact Funct Neurosurg 1999;72:208-18

12. Pahwa R, Lyons KE, Wilkinson SB, et al. Long-term evaluation of deep brain stimulation of the thalamus. J Neurosurg 2006;104:506-12

13. Yamada K, Akazawa K, Yuen S, et al. MR imaging of ventral thalamic nuclei. AJNR Am J Neuroradiol 2010;31:732-35

14. Morel A, Magnin M, Jeanmonod D. Multiarchitectonic and stereotactic atlas of the human thalamus. J Comp Neurol 1997;387:588-630

15. Johansen-Berg H, Behrens TE, Sillery E, et al. Functional-anatomical validation and individual variation of diffusion tractography-based segmentation of the human thalamus. Cereb Cortex 2005;15:31-39

16. Biswal B, Yetkin FZ, Haughton VM, et al. Functional connectivity in the motor cortex of resting human brain using echo-planar MRI. Magn Reson Med 1995;34:537-41

17. Fox MD, Raichle ME. Spontaneous fluctuations in brain activity observed with functional magnetic resonance imaging. Nat Rev Neurosci 2007;8:700-11

18. Zhang D, Snyder AZ, Fox MD, et al. Intrinsic functional relations between human cerebral cortex and thalamus. J Neurophysiol 2008;100:1740-48

19. Zhang D, Snyder AZ, Shimony JS, et al. Noninvasive functional and structural connectivity mapping of the human thalamocortical system. Cereb Cortex 2010;20:1187-94

20. Fair DA, Bathula D, Mills KL, et al. Maturing thalamocortical functional connectivity across development. Front Syst Neurosci 2010;4:10

21. Anderson JS, Ferguson MA, Lopez-Larson M, et al. Topographic maps of multisensory attention. Proc Natl Acad Sci U S A 2010;107:20110-14

22. Anderson JS, Druzgal TJ, Lopez-Larson M, et al. Network anticorrelations, global regression, and phase-shifted soft tissue correction. Hum Brain Mapp 2011;32:919-34. Epub 2010 Jun 9

23. Anderson JS, Ferguson MA, Lopez-Larson M, et al. Reproducibility of functional connectivity measurements in single subjects. AJNR Am J Neuroradiol 2011;32:548-55. Epub 2011 Jan 27

24. Glover GH, Li TQ, Ress D. Image-based method for retrospective correction of physiological motion effects in fMRI: RETROICOR. Magn Reson Med 2000;44:162-67

25. Cox RW. AFNI: software for analysis and visualization of functional magnetic resonance neuroimages. Comput Biomed Res 1996;29:162-73

26. Cordes D, Haughton VM, Arfanakis K, et al. Frequencies contributing to functional connectivity in the cerebral cortex in "resting-state" data. AJNR Am J Neuroradiol 2001;22:1326-33

27. Tzourio-Mazoyer N, Landeau B, Papathanassiou D, et al. Automated anatomical labeling of activations in SPM using a macroscopic anatomical parcellation of the MNI MRI single-subject brain. Neuroimage 2002;15:273-89

28. Maldjian JA, Laurienti PJ, Kraft RA, et al. An automated method for neuroanatomic and cytoarchitectonic atlas-based interrogation of fMRI data sets. $\mathrm{Neu}$ roimage 2003;19:1233-39

29. Fox MD, Snyder AZ, Vincent JL, et al. The human brain is intrinsically organized into dynamic, anticorrelated functional networks. Proc Natl Acad Sci U S A 2005; 102:9673-78

30. Van Dijk KR, Hedden T, Venkataraman A, et al. Intrinsic functional connectivity as a tool for human connectomics: theory, properties, and optimization. J Neurophysiol 2010;103:297-321. Epub 2009 Nov 4 